\title{
Novel MR imaging method - MAVRIC - for metal artifact suppression after joint replacement in musculoskeletal tumor patients
}

Michiro Susa ${ }^{1,2^{*}}$ D, Sota Oguro ${ }^{3}$, Kazutaka Kikuta², Kazumasa Nishimoto ${ }^{2}$, Keisuke Horiuchi ${ }^{2}$, Masahiro Jinzaki ${ }^{3}$, Masaya Nakamura², Morio Matsumoto ${ }^{2}$, Kazuhiro Chiba ${ }^{1}$ and Hideo Morioka ${ }^{2}$

\begin{abstract}
Background: Standard imaging modality for the follow-up after prosthetic replacements for musculoskeletal tumor patients has been conventional radiography. This technique is effective in detecting subtle changes in bone adjacent to metal implants, but in many cases, radiographs do not lead to definitive diagnosis of postoperative adverse events such as acute infection, local recurrence of soft tissue tumor or soft tissue local recurrence of osseous sarcoma. Conventional MRI sequences have not been effective due to metal artifacts. In this study, we tried to elucidate the effectiveness of metal artifact suppression using novel sequence, multiacquisition variable-resonance image combination (MAVRIC), after musculoskeletal tumor surgeries.

Methods: We retrospectively analyzed 5 cases of malignant bone and soft tissue sarcoma patients who were reconstructed with metal prosthesis after wide resection of tumors. Images obtained using MAVRIC and short tau inversion recovery (STIR) were compared side by side. The paired MAVRIC and STIR images were qualitatively compared independently by two specialists for 4 parameters: visualization of bone - implant interface, visualization of surrounding soft tissues, image blurring, and overall image quality. Quantitatively, paired images were reviewed to identify the slice where the metal artifact was maximal, and a region of interest encompassing the implant and surrounding artifact was drawn using Advantage Workstation (GE Healthcare, Japan).

Results: There were no local recurrences that were detected. By utilizing MAVRIC, visualization of the bone - implant interface and visualization of the surrounding soft tissue were significantly improved in MAVRIC compared to STIR. Although blurring was worse on the MAVRIC acquisitions, the overall image quality was still better on MAVRIC. Quantitatively, the area of metal artifact measured using MAVRIC was markedly less compared to STIR $\left(61.4 \mathrm{~cm}^{2}\right.$ vs $135.9 \mathrm{~cm}^{2}$ ).

Conclusion: Despite the relatively small number of cases in the present study, our observation strongly suggests that MAVRIC is able to improve the quality of images by decreasing the artifact caused by endoprosthesis, frequently utilized in reconstruction of musculoskeletal tumor patients. Further installments of conventional imaging sequences with the addition of gadolinium - enhancement will enable increased accuracy in diagnosing local recurrences of sarcoma patients.
\end{abstract}

Keywords: MRI, MAVRIC, Metal artifact

\footnotetext{
* Correspondence: mitchsusa@gmail.com

'Department of Orthopaedic Surgery, National Defense Medical College, 3-2

Namiki, Tokorozawa, Saitama 359-8513, Japan

${ }^{2}$ Department of Orthopedic Surgery, Keio University School of Medicine, 35

Shinanomachi, Shinjuku-ku, Tokyo 160-8582, Japan

Full list of author information is available at the end of the article
} 


\section{Background}

Multimodal therapy for sarcoma patients has improved the prognosis in the past decades, but surgery is still the mainstay of treatment for malignant tumors. Surgery for sarcoma of both bone and soft tissue usually consists of wide resection including the cuff of normal tissue, which leads to large defects. When skeletal structure is compromised, especially near the joint, endoprosthesis is frequently utilized for the reconstruction. Traditionally, radiograph was utilized to detect the change in bone, but it has not enabled the detection of devastating postoperative events such as soft tissue recurrences. The usage of massive metal implants have prevented the usage of magnetic resonance imaging (MRI) which has high spatial resolution and tissue contrasts compared to plain radiographs due to metal artifacts.

Recently, improvements in metal composition of prosthesis [1,2] and artifact-reducing MRI sequences have been reported [3-5]. Multiacquisition variableresonance image combination (MAVRIC) was first reported in 2009, where it uses multiple excitations to excite the overall volume being imaged [3]. MAVRIC excites a series of limited spectral distortion and uses the three-dimensional (3D) spin-echo acquisition to resolve the profile of each excited slice in the region of interest. MAVRIC has been reported to reduce the metal artifacts around smaller metal implants usually reserved for conventional orthopaedic procedure $[4,6,7]$. In this study, we analyzed the effectiveness of MAVRIC for suppression of metal artifacts around larger tumor endoprosthesis for early possible detection of tumor recurrences.

\section{Methods}

Since 2014, 4 megaprosthesis ( 1 total femur, 1 proximal femur, 1 distal femur, and 1 proximal humerus) and 1 augmented proximal femoral reconstruction stem were utilized for reconstructions and underwent MR imaging on a 3T scanner (Discovery 750, GE Healthcare, Japan). Histological diagnosis included 2 osteosarcomas, 2 chondrosarcomas and 1 synovial sarcoma. There were 4 female and1 male. Proximal humeral implant and reconstruction stem were composed from titanium alloy and all other implants were made up of cobalt-chrome. Conventional short tau inversion recovery (STIR) and MAVRIC sequences were acquired in the coronal planes using similar scan parameters and readout bandwidth. MAVRIC sequences were obtained with a 3T scanner (GE SIGNA discovery MR 750, GE Healthcare, Milwaukee, WI) in the supine position. The protocol included coronal, STIR images (TR/TE: 4500-6700/6.3-6.8 ms; inversion time $175 \mathrm{~ms}$; slice thickness $4.5 \mathrm{~mm}$; gap $0 \mathrm{~mm}$; flip angle $65^{\circ}$; field of view $280 \mathrm{~mm}$, matrix $320 \times 320,24$ slices, band width $125 \mathrm{kHz}$, acquisition time from $4 \min 15 \mathrm{~s}$ to
6 min $32 \mathrm{~s}$ ) and coronal proton density weighted images (TR/TE: 2400/6.3-7.1 ms; slice thickness $4.5 \mathrm{~mm}$; gap $0 \mathrm{~mm}$; flip angle $75^{\circ}$; field of view $280 \mathrm{~mm}$, matrix $384 \times$ 320 , 24 slices, band width $125 \mathrm{kHz}$, acquisition time from $4 \min 5 \mathrm{~s}$ to $6 \min 7 \mathrm{~s}$ ) with an 32 - channel torso surface coil. These MR images were retrospectively analyzed between two musculoskeletal tumor specialists (one radiologist and one orthopaedic oncologist) in a matching anatomic plane with the maximal artifact for both qualitative and quantitative variables. Artifact was defined as areas of signal void pile-up or geometric distortion. Images were graded on a five-point scale from -2 to +2 , where lower score suggested better outcome for MAVRIC acquisitions, which was previously reported by Gutierrez et al. [8]. -2 indicate that MAVRIC is significantly better, and -1 meant somewhat better compared to STIR. Readers were blinded as to the type of sequence that they were grading. Quantitatively, plane with the maximal artifact was determined, and region of interest was measured using Advantage Workstation (GE Healthcare, Japan) (Fig. 1). This study was conducted with the approval of Institutional Review Board of Keio University and all participants gave their informed consent to assessing and using their data.

\section{Results}

There were no local recurrences that were detected and no complications such as local hotness during the examination. The quality of images were compared and scored independently between the 2 observers. By utilizing MAVRIC, visualization of the bone - implant interface (average grade: -1.88 ) (Fig. 2) and visualization of the surrounding soft tissue (average grade: -1.75 ) (Fig. 3) were significantly improved in MAVRIC compared to STIR. Although blurring was worse on the MAVRIC acquisitions with lower resolution, lower contrasts and reduced fat saturation (average grade: +1.12) (Fig. 4), the overall image quality was still better on MAVRIC (average grade: -1.88). Quantitatively, the area of metal artifact measured using MAVRIC (range: $43-107 \mathrm{~cm}^{2}$, average: $61.4 \mathrm{~cm}^{2}$ ) was less compared to STIR (range: $76.3-246 \mathrm{~cm}^{2}$, average: $135.9 \mathrm{~cm}^{2}$ ). Clinically, MAVRIC was able to detect fluid collection surrounding the implant in one patient. Although the entire body of the patient was swollen and covered with eruption which was suggestive of toxic shock syndrome, thrombotic thrombocytopenia purpura/hemolytic uremic syndrome, or adult stills syndrome, MAVRIC acquisition revealed fluid collection surrounding the endoprosthesis (Fig. 3b), which lead to local puncture and diagnosis of methicillin-sensitive Staphylococcus aureus infection. The patient underwent irrigation and at the final followup, the patient is well with no symptom. 


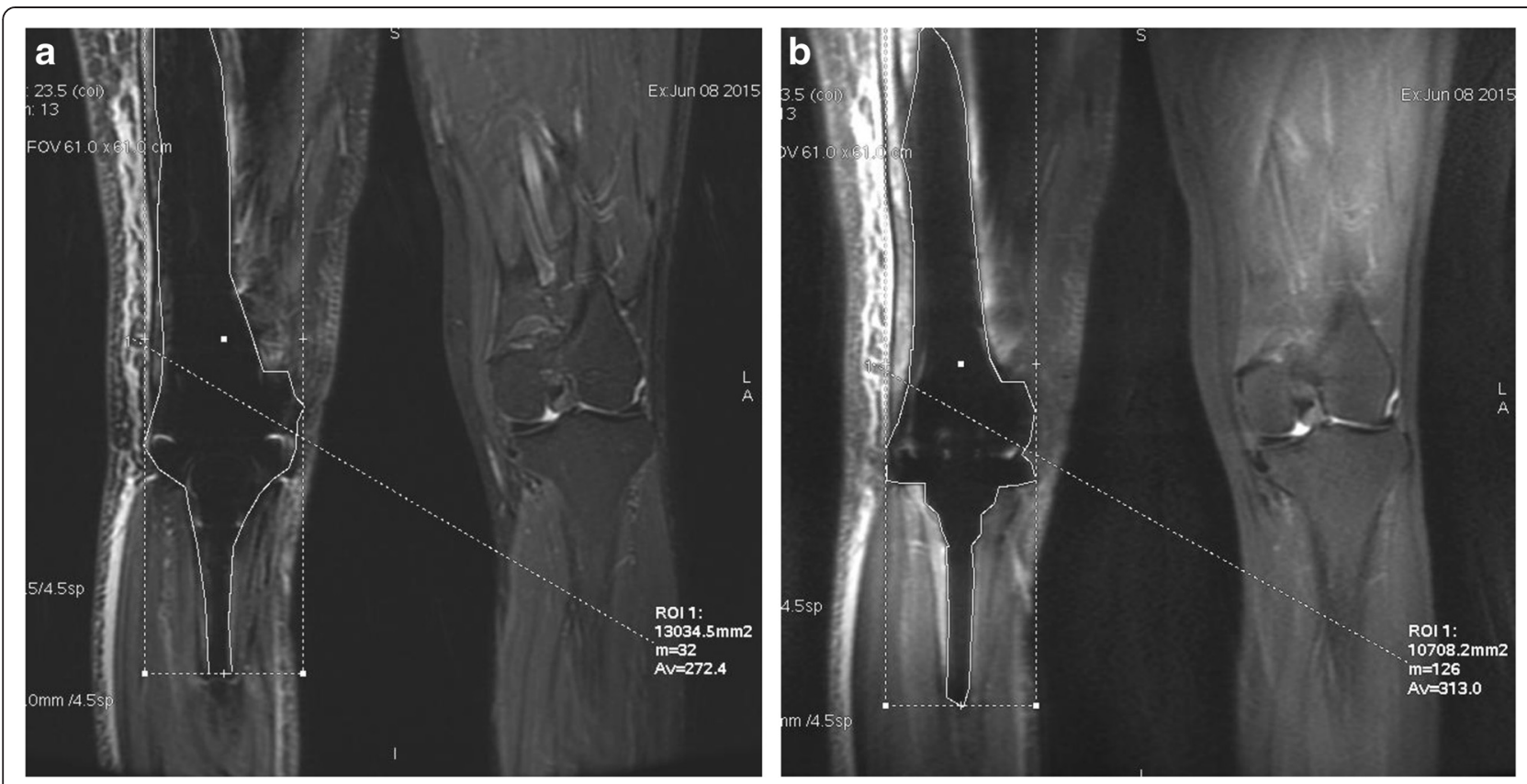

Fig. 1 a. Conventional STIR sequence obained after distal femoral endoprosthesis reconstruction after chondrosarcoma resection in a 46 year - old female. b. MAVRIC image. The maximum area of metal artifact was measured in a matching plane for both images. Area of the artifact was $130.3 \mathrm{~cm}^{2}$ for STIR and $107.0 \mathrm{~cm}^{2}$ for MAVRIC
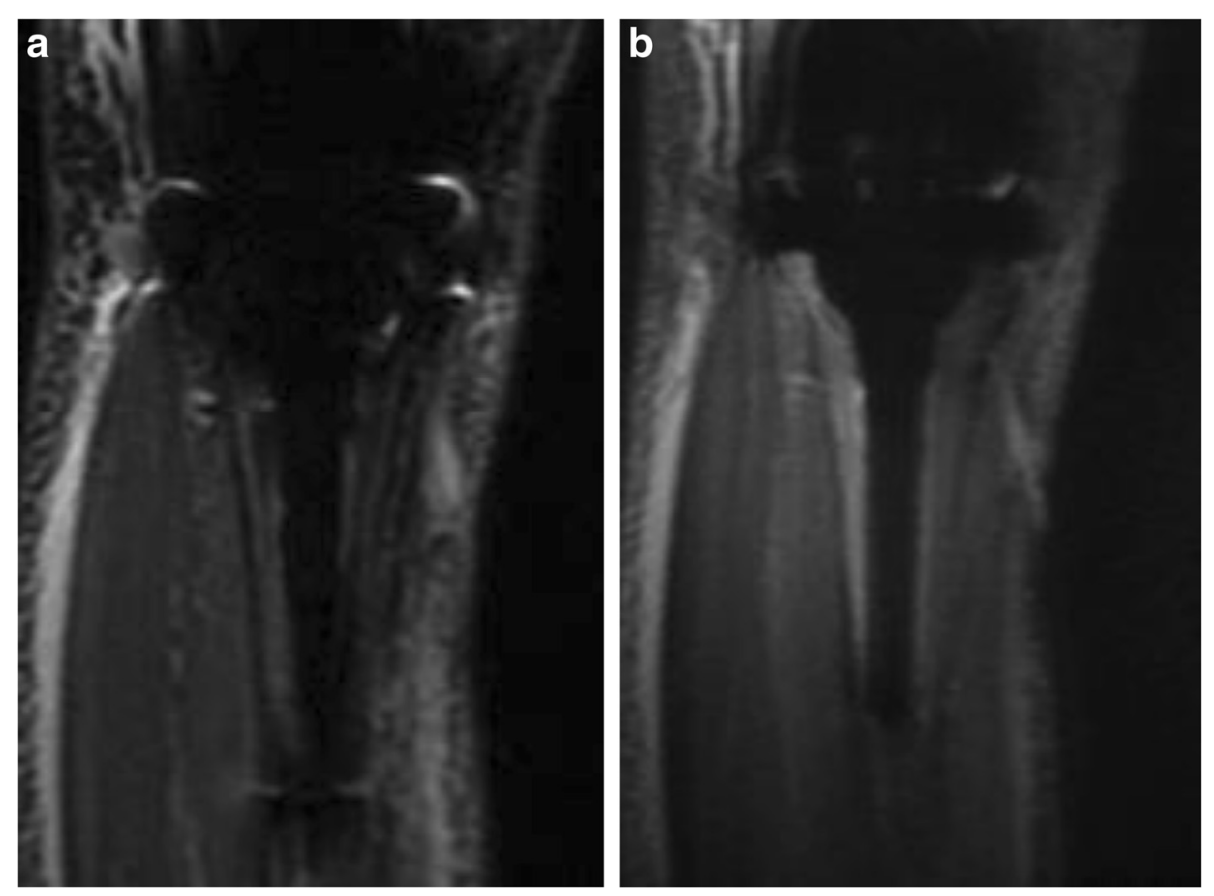

Fig. 2 a. Conventional STIR image and b. MAVRIC image were compared for bone and implant interface. MAVRIC was able to depict the bone immediately adjacent to the endoprosthesis. Additionally, because of the reduced artifact, the prosthesis itself is now visible as signal void especially around the knee 
a

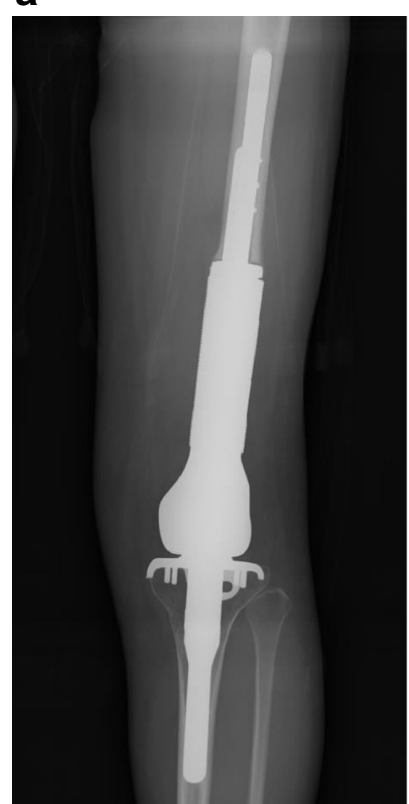

\section{b}

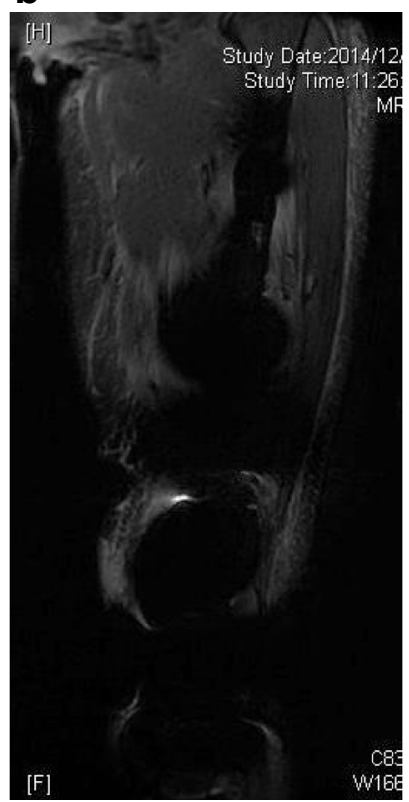

C

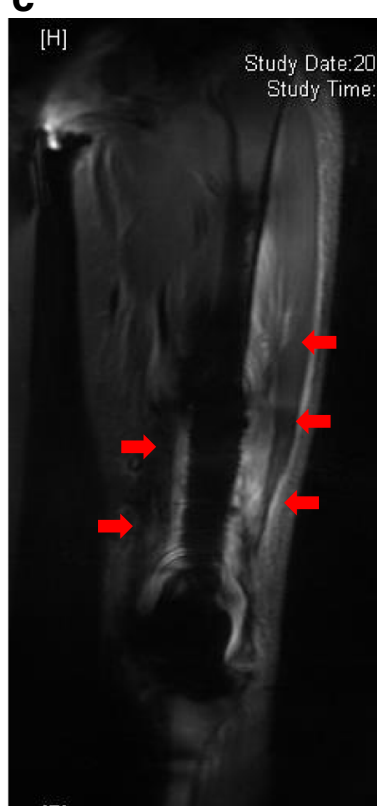

Fig. 3 a. A 27 year - old female received a distal femoral endoprosthetic reconstruction after wide resection of an osteosarcoma 6 years prior to the exmaination. b. Conventional STIR image and c. MAVRIC image were compared for the depiction of surrounding soft tissue adjacent to the endoprosthesis. Joint effusion is observed surrounding the metal implant in MAVRIC image (red arrow). Due to early detection of the periprosthetic infection, thorough irrigation and debriedement was performed, and the patient is well without recurrence

\section{Discussion}

There are many histological subtypes of sarcoma of both bone and soft tissues, and treatment is increasingly becoming case-specific with the advent of genetic testing. Several molecular targeting agents have been reported to improve the prognosis and many more are set to be reported. Although, these medical therapies are important, almost all sarcomas need some kind of surgical intervention to eradicate the tumor. It is especially important to perform a wide resection of the lesion to prevent local
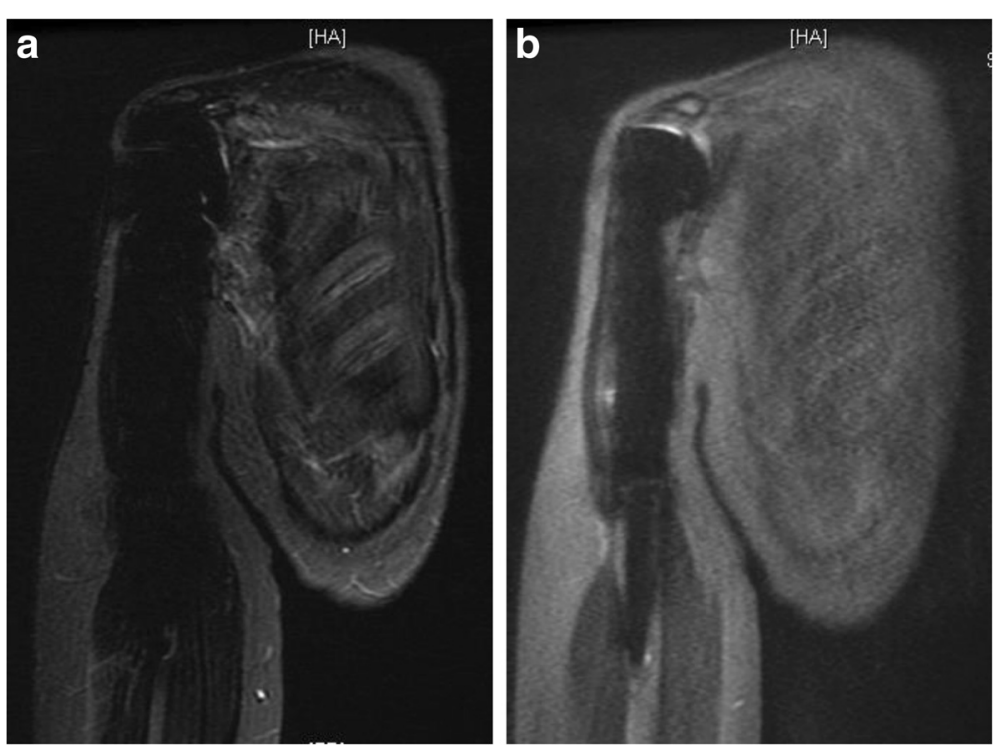

Fig. 4 a. STIR image of a proximal humeral endoprosthetic reconstruction and $\mathbf{b}$. MAVRIC image were compared side by side for the qualitaive assesment of the blurring. Image quality of the MAVRIC is reduced compared to STIR with blurry contours, lower contrast, and lower resolution as depicted by the ribs (average grade: +1.12 ). The fat saturation is clearly reduced with the MAVRIC as seen in the subcutaneous fat tissue 
recurrence from the residual sarcoma cells. To reconstruct large defects, several methods have been proposed including endoprosthesis, allografts, autografts, and recycled bone by pasteurization or liquid nitrogen. When the defect is situated around the joint, endoprosthesis is often utilized due to the poor outcome using other reconstruction methods.

In order to improve the outcome of sarcoma patients, it is important to control the various postoperative adverse events such as acute infection and local recurrence as well as the distant metastasis which is a devastating event both physically and psychologically. Infection leads to multiple salvage operations and delay of chemotherapy which is a poor prognosticator of survival. Local recurrences have been reported to cause higher morbidity due to consequent distant metastasis [9]. One of the reasons for poor outcome after local recurrence is the difficulty of additional surgery. It is difficult to ascertain the tumor infiltration after multiple operations and it is even more difficult after endoprosthesis placement. One of the method recently reported utilize positron emission tomography (PET) - CT to localize the recurrence in the vicinity of metal implants [10]. We have also reported on the possibility of local recurrence detection using PET-CT, where SUV $V_{\max }$ of 5.0 or greater might differentiate between tumor and fibrosis (data not shown). But, utilization of CT still causes artifacts due to beam hardening which pose significant challenge in delineating the tumor for successful surgery. Novel method for early diagnosis of postoperative adverse event is imperative to improve the outcome of sarcoma patients.

MRI has improved the prognosis of sarcoma patients by accurately detecting the localization and spread of the tumor prior to operation. It has the highest spatial resolution and tissue contrasts compared to other imaging modalities, but metal implants after surgery have impeded its accuracy by causing artifacts that results from metal disturbing the main magnetic field and inducing strong and spatially - varying local gradients [11]. MRI implementation on tumor endoprosthesis including expandable prosthesis has been reported to be safe without local hotness or unintentional lengthening [12]. MAVRIC and the slice encoding for metal artifact attenuation technique has shown promising results by reducing artifacts. MAVRIC has been reported in several reports after orthopaedic implant surgery as a possible solution for suppression of artifacts $[4,6,7,13]$. MAVRIC has been able to detect joint abnormalities such as joint effusion and bursitis in painful hip [4] and shoulder [7], and showed clinical relevance after total knee replacements [14]. Results from this study also demonstrate that MAVRIC correction for metal induced artifacts improved postoperative visualization around the endoprosthesis. Fortunately, no local recurrence was detected in this case series, but imaging finding of local fluid collection was confirmed by subsequent invasive treatment in one case.

A limitation of this study is its relatively small sample size, which is consistent with other past small case series. Although the preparation of images is still time consuming, it is well suited for dedicated application in areas under suspicion. Further improvement, such as the introduction of MAVRIC - T1WI will enable the use of gadolinium - enhancement and increase the sensitivity for small lesions near the metal implants.

\section{Conclusion}

Several variations of metal artifact suppression technique such as FSE, patient positioning, and use of high bandwidth have been reported [5, 15-17], but there is still significant challenge in detecting small lesions near metal implants. MAVRIC significantly improved the image quality and has the potential to improve patient managements. However, further prospective randomized studies are needed to establish the optimum use of MAVRIC for early diagnosis of local recurrences after sarcoma surgeries.

\section{Competing interests}

The authors declare that there is no conflict of interests.

\section{Authors' contributions}

MS carried out the data analysis and manuscript writing. SO and KK carried out the collection and assembly of data. $\mathrm{KN}$ and $\mathrm{KH}$ contributed to the manuscript writing. MJ, MN, KC and MM provided administrative support. HM was responsible for the provision of study material of patients and final approval of the manuscript. All authors read and approved the final manuscript.

\section{Author details}

'Department of Orthopaedic Surgery, National Defense Medical College, 3-2 Namiki, Tokorozawa, Saitama 359-8513, Japan. ${ }^{2}$ Department of Orthopedic Surgery, Keio University School of Medicine, 35 Shinanomachi, Shinjuku-ku, Tokyo 160-8582, Japan. ${ }^{3}$ Department of Diagnostic Radiology, Keio University School of Medicine, 35 Shinanomachi, Shinjuku-ku, Tokyo 160-8582, Japan.

Received: 25 September 2015 Accepted: 1 December 2015 Published online: 04 December 2015

\section{References}

1. Wang JC, Yu WD, Sandhu HS, Tam V, Delamarter RB. A comparison of magnetic resonance and computed tomographic image quality after the implantation of tantalum and titanium spinal instrumentation. Spine (Phila Pa 1976). 1998;23(15):1684-8.

2. Lee MJ, Kim S, Lee SA, Song HT, Huh YM, Kim DH, et al. Overcoming artifacts from metallic orthopedic implants at high-field-strength MR imaging and multi-detector CT. Radiographics. 2007;27(3):791-803.

3. Koch KM, Lorbiecki JE, Hinks RS, King KF. A multispectral three-dimensional acquisition technique for imaging near metal implants. Magn Reson Med. 2009;61(2):381-90.

4. Kretzschmar M, Nardo L, Han MM, Heilmeier U, Sam C, Joseph GB, et al. Metal artefact suppression at 3 T MRI: comparison of MAVRIC-SL with conventional fast spin echo sequences in patients with Hip joint arthroplasty. Eur Radiol. 2015;25(8):2403-11.

5. Vandevenne JE, Vanhoenacker FM, Parizel PM, Butts Pauly K, Lang RK. Reduction of metal artefacts in musculoskeletal MR imaging. JBR-BTR. 2007;90(5):345-9. 
6. Choi SJ, Koch KM, Hargreaves BA, Stevens KJ, Gold GE. Metal artifact reduction with MAVRIC SL at 3-T MRI in patients with hip arthroplasty. AJR Am J Roentgenol. 2015;204(1):140-7.

7. Hayter CL, Koff MF, Shah P, Koch KM, Miller TT, Potter HG. MRI after arthroplasty: comparison of MAVRIC and conventional fast spin-echo techniques. AJR Am J Roentgenol. 2011;197(3):W405-11.

8. Gutierrez LB, Do BH, Gold GE, Hargreaves BA, Koch KM, Worters PW, et al. MR imaging near metallic implants using MAVRIC SL: initial clinical experience at 3T. Acad Radiol. 2015;22(3):370-9.

9. Trovik CS, Gustafson P, Bauer HC, Saeter G, Klepp R, Berlin O, et al. Consequences of local recurrence of soft tissue sarcoma: 205 patients from the Scandinavian Sarcoma Group Register. Acta Orthop Scand. 2000;71(5):488-95.

10. Gelfand MJ, Sharp SE. [F-18]2-fluoro-2-deoxyglucose (FDG) positron emission tomography after limb salvage surgery: post-surgical appearance, attenuation correction and local complications. Pediatr Radiol. 2015;45(8):1182-8

11. Wendt 3rd RE, Wilcott 3rd MR, Nitz W, Murphy PH, Bryan RN. MR imaging of susceptibility-induced magnetic field inhomogeneities. Radiology. 1988;168(3):837-41.

12. Ogg RJ, McDaniel CB, Wallace $D$, Pitot $P$, Neel MD, Kaste SC. MR safety and compatibility of a noninvasively expandable total-joint endoprosthesis. Magn Reson Imaging. 2005;23(7):789-94.

13. Sutter R, Ulbrich EJ, Jellus V, Nittka M, Pfirrmann CW. Reduction of metal artifacts in patients with total hip arthroplasty with slice-encoding metal artifact correction and view-angle tilting MR imaging. Radiology. 2012;265(1):204-14.

14. Chen CA, Chen W, Goodman SB, Hargreaves BA, Koch KM, Lu W, et al. New MR imaging methods for metallic implants in the knee: artifact correction and clinical impact. J Magn Reson Imaging. 2011;33(5):1121-7.

15. Butts K, Pauly JM, Gold GE. Reduction of blurring in view angle tilting MRI. Magn Reson Med. 2005;53(2):418-24.

16. Cho ZH, Kim DJ, Kim YK. Total inhomogeneity correction including chemical shifts and susceptibility by view angle tilting. Med Phys. 1988;15(1):7-11.

17. Walde TA, Weiland DE, Leung SB, Kitamura N, Sychterz CJ, Engh Jr CA, et al. Comparison of CT, MRl, and radiographs in assessing pelvic osteolysis: a cadaveric study. Clin Orthop Relat Res. 2005;437:138-44.

\section{Submit your next manuscript to BioMed Central and we will help you at every step:}

- We accept pre-submission inquiries

- Our selector tool helps you to find the most relevant journal

- We provide round the clock customer support

- Convenient online submission

- Thorough peer review

- Inclusion in PubMed and all major indexing services

- Maximum visibility for your research

Submit your manuscript at www.biomedcentral.com/submit
( BioMed Central 